\title{
Sonic Boom Minimization Using Improved Linearized Tools and Probabilistic Propagation
}

\author{
Sriram. K. Rallabhandi* and Dimitri N. Mavris ${ }^{\dagger}$ \\ Georgia Institute of Technology, Atlanta, GA 30332
}

\begin{abstract}
Sonic boom modelling is multidisciplinary involving aerodynamic and aero-acoustics analyses. The near field pressure signature is first obtained using either linearized or non-linear methods. This is then converted into a F-function, which is then propagated to the ground using aero-acoustic routines. Existing linearized methods operate on simple approximations of true geometry. Using improved linearized tools that operate on unstructured water-tight geometries, the accuracy and efficacy of shape optimization can be greatly improved. The sonic boom minimization technique is reformulated as an optimization problem and boom propagation is carried out in a probabilistic fashion. A bi-level reverse optimization is conducted to design aircraft to meet low sonic boom requirements under atmospheric uncertainty.
\end{abstract}

\section{Nomenclature}

$\begin{array}{ll}\alpha_{y} & \text { Non-linear advance of acoustic rays } \\ A^{2} & \text { Anderson-Darling test statistic } \\ B & \text { Rise slope in F-function } \\ b_{1} & \text { Input layer bias vector } \\ b_{2} & \text { Output layer bias vector } \\ H, C, D, \lambda, y_{r} \text { Parameters associated with F-function } \\ p r / p f & \text { Ratio of rear to front shock strength } \\ S & \text { Slope of balancing line in F-function } \\ V & \text { Hidden layer network weights } \\ W & \text { Output layer network weights } \\ y_{f} & \text { Bluntness parameter }\end{array}$

\section{Introduction and Motivation}

$\mathrm{T}_{\mathrm{N}}^{\mathrm{N}}$ any design environment, simple theories are initially applied to the proposed concept and advanced computational aerodynamic packages are utilized later in the design stages. Most of the advanced analyses are multidisciplinary in nature with iterations between aerodynamics, structures, flight mechanics and propulsion. Multidisciplinary analysis is computationally very intensive and is dependent upon the concept provided from the initial stages. Thus, one cannot overlook the role played by the conceptual design. Conceptual design is also important from another perspective. The designer has a lot of design freedom in the conceptual stages as shown in Figure 1 This figure from Mavris and Delaurentis, ${ }^{1}$ shows the comparison of design freedom and knowledge available at various stages of design between today's framework and a notional future framework. As the design proceeds through various stages, the design space shrinks to eventually become the final design. The designer has to take advantage of the huge design space upfront to avoid serious and costly alterations in design during the later stages.

\footnotetext{
${ }^{*}$ Graduate Research Assistant, Aerospace Systems Design Lab, AIAA Student member.

${ }^{\dagger}$ Director and Boeing Professor of Advanced Aerospace Systems Analysis, Aerospace Systems Design Lab, Associate Fellow AIAA
} 


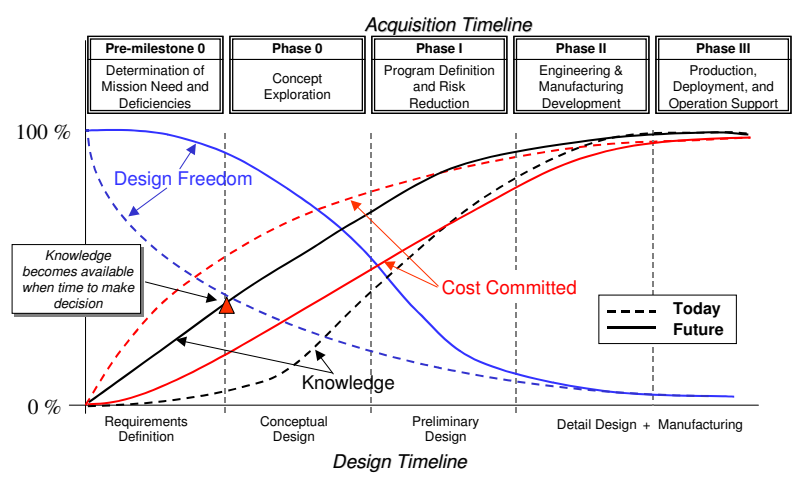

Figure 1. Different stages of design.

According to market studies conducted by various organizations, ${ }^{2}$ there is a need for an efficient, low noise commercial airplane that could travel at supersonic speeds over land. To achieve this, numerous technical challenges have to be overcome. The most important of these challenges are sonic boom minimization, engine emissions and airport noise. ${ }^{3}$ Since smaller aircraft have lesser weight and thus produce lower sonic boom levels, recent research has concentrated on designing small supersonic business jets. A successful design for such a small aircraft would then serve as a stepping stone for future commercial supersonic research. With increased importance being given to the conceptual design, better aerodynamic analysis in the early phases of design could lead to significant improvements in the overall design cycle of the aircraft. Better aerodynamic analyses usually trickles down to better geometry representation and discretization as these are the primary prerequisites to run high fidelity aerodynamic analysis.

\section{Geometry generation and discretization}

An efficient shape parameterization strategy is a prerequisite for performing aerodynamic shape optimization. Geometry generation is a key issue in shape optimization studies. Various techniques have been introduced in the past to create efficient parametric geometries. Bloor and Wilson $\underline{4}$ introduced a partial differential equation approach to obtain arbitrary aircraft configurations by solving a bi-harmonic partial differential equation (PDE). Smith et.al ${ }^{[5}$ extended the PDE approach to generate arbitrary configurations along with volume grid generation and grid sensitivity. Various geometry generation tools and their impor-

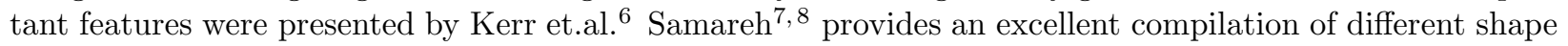
parameterization techniques.

The above techniques, though very useful to create mathematically closed surfaces in further stages of design, consume a significant set-up and computational time. What is needed in conceptual design is a technique by which many geometries can be analyzed in a quick and efficient manner to obtain the same level of fidelity achieved by the tools mentioned in the previous paragraph. Importance has to be given to automation and computational time. A MATLAB based geometry generation and discretization method has been demonstrated by the authors ${ }^{9}$ to create water-tight geometries quickly and efficiently.

The idea is to use variables to control the shape as well as the configuration of the aircraft. The configuration variables are discrete and different values for these produce different types of components as shown in table 1 As can be seen from this table, various shapes are already programmed into the geometric tool and this results in a wide variety of configurations that can be generated. For example, depending on the value of discrete wing parameter, the wing geometry can be a conventional, delta, double-delta, multisection or a swing-wing design. Canard, conventional or T-tail geometries and configurations with various engine configurations can also be generated. From table [1 if all components have to exist, there could be $5 \times 6 \times 3 \times 1 \times 4=360$ discrete types of configurations. In addition, within each configuration, there are various continuous parameters to define the shape of each component.

Table 2 presents some of the important continuous parameters that determine the shape of the aircraft. Included here are various planform parameters, control points for NURBS surfaces and bezier curves. Fuselage shapes produced by the formulation include axisymmetric and non-axisymmetric fuselages which are pointed or blunt or area-ruled. Wing shape parameters include twist, camber, control points for leading edge 


\begin{tabular}{|c|c|c|c|c|c|c|c|}
\hline \multirow[t]{2}{*}{ Component } & \multicolumn{7}{|c|}{ Component type parameter } \\
\hline & 0 & 1 & 2 & 3 & 4 & 5 & 6 \\
\hline Fuselage & No Fuselage & $\begin{array}{l}\text { Nose specified } \\
\text {-bezier curve }\end{array}$ & $\begin{array}{c}\text { Full Fuselage } \\
\text { - bezier curves }\end{array}$ & $\begin{array}{l}\text { NURBS nose, } \\
\text { rest-bezier curves }\end{array}$ & $\begin{array}{c}\text { Fuselage in } \\
\text { cross-sections }\end{array}$ & $\begin{array}{c}\text { Area ruled } \\
\text { Fuselage }\end{array}$ & \\
\hline Wing & No wing & $\begin{array}{l}\text { Conventional } \\
\text { wing }\end{array}$ & $\begin{array}{r}\text { Delta } \\
\text { wing }\end{array}$ & $\begin{array}{l}\text { Double delta } \\
\text { wing }\end{array}$ & $\begin{array}{c}\text { Concorde like } \\
\text { multi-section wing }\end{array}$ & Swing-wing & $\begin{array}{l}\text { Raytheon like } \\
\text { multi-section wing }\end{array}$ \\
\hline H-Tail & No H-Tail & Canard & Conventional & T-tail & & & \\
\hline V-Tail & No V-Tail & Conventional & & & & & \\
\hline Engine & No Engines & $\begin{array}{l}\text { Wing mounted, } \\
2 \text { below wing }\end{array}$ & $\begin{array}{l}\text { Fuselage } \\
\text { mounted }\end{array}$ & $\begin{array}{l}\text { Wing mounted, } \\
2 \text { above wing }\end{array}$ & $\begin{array}{l}\text { Wing mounted, } \\
4 \text { below wing }\end{array}$ & & \\
\hline
\end{tabular}

Table 1. Discrete parameters

bezier curve and various other parameters. Other parameters are simply dimensions and planform locations of components. The maximum number of parameters used to create a single configuration is 75 .

\begin{tabular}{|c|c|}
\hline Component & Important continuous parameters \\
\hline Fuselage & $\begin{array}{r}\text { Length, Maximum diameter, Max. diameter location, bezier control points for nose, mid-section and aft region, } \\
\text { Camber, Camber location, blunt-ness parameter, parameters for non-axisymmetric nose section }\end{array}$ \\
\hline Wing & $\begin{array}{r}\text { Wing location, } t / c, \text { camber, camber location, twist distribution, dihedral, } \\
\text { Bezier control points for leading edge,sweep, span }\end{array}$ \\
\hline H-Tail & Aspect ratio, taper ratio, sweep, t/c, longitudinal location, vertical location \\
\hline V-Tail & Aspect ratio, taper ratio, sweep, t/c, longitudinal location \\
\hline Engine & Engine location, radius, hub to tip ratio, engine length \\
\hline
\end{tabular}

Table 2. Important continuous parameters

With this parameterization strategy a wide design space of geometries can be generated. Once a geometry has been created, it should be discretized for numerical analysis. The shapes obtained by the design process are of not much use for further analysis if these shapes cannot be translated into a CAD definition for manufacturing. Ability to manufacture should be induced into the design process right from the conceptual level. This could reduce the time during design iteration and thus reduce the total life cycle cost of the final end product. It is therefore essential to create an integrated design procedure, where generated configurations are easily and automatically translated into water-tight CAD geometries. Apart from manufacturing, a CAD definition provides a common geometry format for various analyses and disciplines.

\section{Traditional and improved near field prediction tools}

It is known that the main components of sonic boom prediction are near field aerodynamic analysis involving estimation of equivalent area due to volume and lift 10 and far field acoustic analysis involving pressure propagation through the atmosphere. In the conceptual design stages, the equivalent area due to volume and wave drag are obtained using AWAVE ${ }^{[1]}$ ALIFT ${ }^{[12]}$ has been traditionally used for area due to lift estimation. The limitations of these tools have been previously discussed by the authors $[9$

After the geometry has been created and discretized, modified linearized analysis tools are required which accept the new geometry definition to produce required aerodynamic output. In this work, linearized analysis is used, although the geometry discretization is amenable to run CFD analysis. An improvement to the conceptual tools for sonic boom analysis could be accomplished either by using high fidelity analysis or by providing an improved geometry definition to the analyses. While using better geometry input with low fidelity analyses is not as accurate as using high fidelity analysis, it is certainly superior to using low fidelity analyses over a poor geometric description. Based on the limitations of the existing linearized codes, improved linearized tools that operate on the unstructured geometries from section II have been developed and demonstrated. ${ }^{[]}$For the equivalent area due to volume contribution, proposed method uses efficient 
geometric algorithms to obtain the true Mach-plane intercepted area. The results have been thoroughly validated. The equivalent area due to lift estimation routines have been modified to run on the geometry created. The designer could replace the lift analysis by a generalized vortex lattice method, panel method or a full blown computational fluid dynamics simulation depending on the level of fidelity desired.

Using the discretized geometry format, the wave drag of the aircraft can be computed by performing surface boolean operations $\frac{13}{13}$ Table 3 presents the wave drag numbers associated with a simple wing-bodycanard geometry at Mach number of 1.4. As can be seen from this table, AWAVE highly over-predicts the wave drag values whereas the improved tool produces wave drag numbers closer to the CFD analysis result.

\begin{tabular}{|c|c|c|c|}
\hline Using Improved tools & Using AWAVE & AWAVE with wing truncation & Using CFD \\
\hline$\frac{D_{w}}{q}=3.0798$ & $\frac{D_{w}}{q}=5.1879$ & $\frac{D_{w}}{q}=4.8039$ & $\frac{D_{w}}{q}=2.76$ \\
\hline
\end{tabular}

Table 3. Wave drag comparison using various methods

\section{Probabilistic propagation}

A propagation model is needed to propagate the aerodynamic near field pressure signature to the ground. Traditional propagation models assume standard atmospheric properties to obtain the pressure and temperature values at different altitudes which are then used to obtain the sonic boom pressure signature on the ground. Linearized propagation models like ARAP 14 are approximations of the true pressure propagation. Effects like atmospheric absorption, molecular relaxation, turbulence and anomalies in temperature and wind profiles influence the ground pressure signature. Unfortunately, none of these effects are modelled in a conceptual propagation tool like ARAP. An improved propagation code, $\mathrm{PCBOOM}, \frac{15}{15}$ performs a three dimensional propagation of the near field pressure signature and includes the effect of molecular relaxation to a certain extent. There is a need, at the conceptual level, to include the effect of anomalies present in temperature and wind profiles. Atmospheric fluctuations could cause variations in the pressure signature on the ground. PCBOOM can inherently model user input atmospheric profiles. To complement that, ARAP code has also been modified to account for these variations in the atmospheric parameters. In order to model the temperature fluctuations, normal distributions are placed around selected parameters like lapse rates and the heights which separate the atmospheric layers to yield varying temperature profiles. The effect of atmospheric fluctuations is performed in the following way. Given the area distribution or the F-function, the propagation analysis is run for a fixed number of times with varying temperature profiles. The perceived loudness values for these cases are then used to fit a distribution using Anderson-Darling test statistic as explained below.

\section{A. Anderson-Darling test statistic}

The Anderson-Darling test is one of the most powerful and important goodness-of-fit tests in the statistical literature especially for small sample sizes. This test is a modification of the Kolmogorov-Smirnov test in that it weighs the tails more heavily and utilizes a hypothesized distribution resulting in a better goodness-of-fit test. Using the sample points, the parameters of the hypothesized distribution are estimated. Then a critical value of the test statistic corresponding to the hypothesized distribution is determined. Depending on the values of the test statistic and the critical values, the hypothesized distribution is accepted or rejected. The Anderson-Darling test statistic is defined in equations 1 and 2 for a normal distribution.

$$
A^{2}=-n-S
$$

where

$$
S=\sum_{i=1}^{N} \frac{(2 i-1)}{N}\left[\ln \left(F_{0}\left(x_{i}\right)\right)+\ln \left(1-F_{0}\left(x_{n+1-i}\right)\right)\right]
$$

If the mean and variance have to be estimated using the same data used for the test, then the test statistic 
is modified according to the equation 3

$$
A^{2}=A^{2} \times\left(1+\frac{4}{N}+\frac{25}{N^{2}}\right)
$$

The critical value for a normal distribution is given by equation 4

$$
C V=0.752 /\left(1+\frac{0.75}{N}+\frac{2.25}{N^{2}}\right)
$$

Now if $A^{2}>C V$, then the hypothesized distribution is rejected as not fitting the sample points. The critical and test statistic values are different for various distributions and is explained in detail in the RAC $\underline{16}$ paper. In this study, Anderson-Darling test has been used to accept or reject 4 distributions, normal, log-normal, weibull and exponential, due to their frequent occurrence in many reliability studies.

After the distribution of the perceived loudness level has been obtained using the Anderson-Darling test, a cumulative probability function for that distribution is obtained. A value corresponding to the $95 \%$ probable value is then used as the perceived loudness level, PLdB, as shown in figure 2. The designer could choose the number of samples to use in the Anderson-Darling test. The higher the number of samples, the closer one can get to the actual CDF.

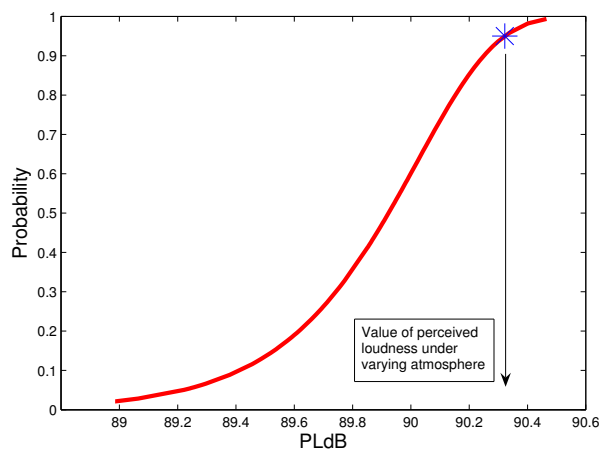

Figure 2. The perceived loudness level from CDF

\section{Method for Sonic Boom minimization}

Sonic boom minimization is one of the core issues of research at many academic institutions and government organizations. The most important issue in minimization studies is to choose a criteria to minimize the ground signature. There is no single standard objective that is used for boom optimization. Various researchers have used one or more of the important quantities associated with a ground pressure signature. A few of these quantities are initial shock pressure rise, maximum overpressure, the time taken to reach the maximum overpressure and the impulse or energy contained in the signature. Boom minimization theory of SeebassGeorge ${ }^{[17}$ and extension by Darden, $\frac{18}{18}$ henceforth referred to as SGD, develops expressions for the near field signature which minimize one or more of the above parameters. This theory provides low boom constraints which are then used as guidelines to drive the optimizer to achieve those near field values by changing the shape of the aircraft.

Recent research shows that, perhaps, the most important parameter that should be used for minimization is the loudness level of the pressure signature that is perceived by humans and structures. Supersonic flight over land would be possible if the noise generated does not have a significant effect on humans and does not cause damage to buildings. The existing minimization theory does not provide lower bounds for perceived loudness, rather it just provides lower bounds for pressure perturbations. Minimizing overpressure or shock pressure rise does not necessarily minimize the perceived loudness and therefore the near field signature predicted by the existing theory may not yield a signature of minimum loudness. Asymmetry of the signature also has an effect on the loudness as studied by Leatherwood ${ }^{19}$ and is implemented in this study. In this section, the SGD theory equations are simplified and recast as a set of two simultaneous equations and an efficient solution strategy is suggested. 
In SGD theory, the coefficients of the F-function are obtained to satisfy certain conditions imposed on the signature. This theory obtains the equivalent area distribution that minimizes the overpressure or the initial shock pressure rise. An efficient and automated solution of SGD theory equations required for aircraft conceptual design is presented here. The F-function is assumed to be of the form shown in equation 5 .

$$
F(y)= \begin{cases}\frac{2 y H}{y_{f}} & 0 \leq y \leq y_{f} / 2 \\ C\left(\frac{2 y}{y_{f}}-1\right)-H\left(\frac{2 y}{y_{f}}-2\right) & y_{f} / 2 \leq y \leq y_{f} \\ B\left(y-y_{f}\right)+C & y_{f} \leq y \leq \lambda \\ B\left(y-y_{f}\right)-D & \lambda \leq y \leq l\end{cases}
$$

The known parameters are $y_{f}, l, M, W$ and $p r / p f$. Based on the Seebass-George-Darden 1718 relations, the following equations can be written.

$$
\begin{gathered}
\int_{l}^{y_{r}} F(y) d y=\frac{-2}{\pi} \int_{0}^{l} F(x) \tan ^{-1}\left(\sqrt{\frac{y_{r}-l}{l-x}}\right) d x=\frac{1}{2}\left[B\left(l-y_{f}\right)-D+F\left(y_{r}\right)\right]\left(y_{r}-l\right) \\
\frac{p_{f}}{p_{r}}=\frac{C}{D-B\left(l-y_{f}\right)+F\left(y_{r}\right)} \\
F\left(y_{r}\right)=S\left(y_{r}-l\right)+B\left(l-y_{f}\right)-D \\
\int_{0}^{y_{f}} F(y) d y=\frac{\alpha_{y_{f}}}{2} C \\
\int_{l}^{y_{r}} F(y) d y=\frac{1}{2}\left[B\left(l-y_{f}\right)-D+F\left(y_{r}\right)\right]\left(y_{r}-l\right) \\
F(y)=-\frac{1}{\pi(y-l)^{1 / 2}} \int_{0}^{l} \frac{(l-\xi)^{1 / 2}}{(y-\xi)} F(\xi) d \xi
\end{gathered}
$$

The purpose of this exercise is to determine the unknowns C,D,H, $\lambda$ and $y_{r}$ given the Mach number, altitude, length and gross weight. Using the geometric acoustics techniques, closed form expressions involving integrals can be used to calculate the value of $\mathrm{S}$ as shown in equation 12

$$
S=-\frac{\sqrt{2 \beta}}{\Gamma M_{h}^{3} \int_{0}^{h} \frac{p_{h}}{p} \sqrt{\frac{\rho a_{h}}{\rho_{h} a}} \sqrt{\frac{A_{h}}{z_{h} A}} \frac{M}{\beta} d z}
$$

where,

$$
\frac{A_{h}}{z_{h} A}=\left[M_{h} \sqrt{\left(1-\frac{1}{M_{z}^{2}}\right)} \int_{0}^{z} \frac{1}{\sqrt{\left(M_{z}^{2}-1\right)}} d z\right]^{-1}
$$

The non-linear advance can be calculated from equation 14 by performing numerical integration.

$$
\alpha_{y}=-\frac{\Gamma M_{h}^{3} F(y)}{\sqrt{2 \beta}} \int_{0}^{z} \frac{p_{h}}{p} \sqrt{\frac{\rho a_{h}}{\rho_{h} a}} \sqrt{\frac{A_{h}}{z_{h} A}} \frac{M}{\beta} d z
$$

Using the supplied values of $h, M, l$ and $G W$, the slope of the balancing can be calculated using equations 12 through 14 The slope of the front balancing line, S, is proportional to the reciprocal of the non-linear advance at any point of the signal. Therefore, equation 12 can also be casted as shown in equation 15 . Equation [16 gives the value of the non-linear advance at $y_{f}$ in the near field signature using equations 7 and [8.

$$
S=\frac{F(y)}{\alpha_{y}}=\frac{C}{\alpha_{y_{f}}}
$$




$$
\alpha_{y_{f}}=\left(y_{r}-l\right) \frac{P_{r}}{P_{f}}
$$

Equation 17 is then obtained as a function of $\alpha_{y_{f}}$ which in turn is a function of $y_{f}$.

$$
C=\frac{2 H y_{f}}{\left(2 \alpha_{y_{f}}-y_{f}\right)}
$$

Using equations [15 and 17 a quadratic equation in $\alpha_{y_{f}}$ can be obtained. The negative root is extraneous and the positive root is taken to be actual advance because the advance cannot be negative. The quadratic can be solved for $\alpha_{y_{f}}$ in terms of $y_{f}, S$ and $H$. The solution is shown in equation 18

$$
\alpha_{y_{f}}=\frac{y_{f} S+\sqrt{y_{f}^{2} S^{2}+16 H y_{f} S}}{4 S}=\left(y_{r}-l\right) \frac{P_{r}}{P_{f}}
$$

From equation 18 $\mathrm{H}$ can be solved in terms of the unknown parameter $y_{r}$ and is given in equation 19

$$
H=\frac{S\left(y_{r}-l\right)^{2}\left(\frac{P_{f}}{P_{r}}\right)^{2}}{y_{f}}-\frac{S\left(y_{r}-l\right) \frac{P_{f}}{P_{r}}}{2}
$$

With $H, \alpha_{y_{f}}$ known in terms of the unknown $y_{r}$, equation 19 can now be used to obtain C in terms of $y_{r}$ by substituting in equation 17 and is given in equation 20

$$
C=\frac{2 S\left(y_{r}-l\right)^{2}\left(\frac{P_{f}}{P_{r}}\right)^{2}-S y_{f}\left(y_{r}-l\right) \frac{P_{f}}{P_{r}}}{2\left(y_{r}-l\right) \frac{P_{f}}{P_{r}}-y_{f}}
$$

The first step is the assumption that minimum pressure disturbances on the ground are theoretically achieved when the volume contribution is absent. In other words, only lift contribution needs to be considered. In such a case, the equivalent area due to lift is as given in equation 21

$$
A_{e}(l)=\frac{\beta W}{\rho U^{2}}=4 \int_{0}^{l} F(y) \sqrt{(l-y)} d y
$$

The above integral can be split into 4 different intervals and carry out the integration. Let $l-\xi=x^{2}$. The above integral reduces to

$$
\begin{aligned}
& A_{e}(l)=\frac{4 H}{y_{f}} \int_{\sqrt{l}}^{\sqrt{l-\frac{y_{f}}{2}}} x^{2}\left(x^{2}-l\right) d x+\frac{4 C}{y_{f}} \int_{\sqrt{l-\frac{y_{f}}{2}}}^{\sqrt{l-y_{f}}} x^{2}\left(4\left(x^{2}-l\right)+2 y_{f}\right) d x-\frac{4 H}{y_{f}} \int_{\sqrt{l-\frac{y_{f}}{2}}}^{\sqrt{l-y_{f}}} x^{2}\left(4\left(x^{2}-l\right)+4 y_{f}\right) d x \\
& +4 \int_{\sqrt{l-y_{f}}}^{\sqrt{l-\lambda}} 2 x^{2}\left(B\left(x^{2}+y_{f}-l\right)-C\right) d x+4 \int_{\sqrt{l-\lambda}}^{0} 2 x^{2}\left(B\left(x^{2}+y_{f}-l\right)+D\right) d x
\end{aligned}
$$

The following relations are assumed to simplify the equations.

$$
\beta=\sqrt{l}, \alpha=\sqrt{l-\frac{y_{f}}{2}}, \gamma=\sqrt{l-y_{f}}, \delta=\sqrt{l-\lambda}
$$

Carrying out the integration, the expression for the equivalent area due to lift with the assumed form of the F-function is given equation 24 This equation can then be used to solve for $D$ in terms of $y_{r}$ and $\lambda$ and the expression is shown in equation 25 .

$$
\begin{aligned}
& A_{e}(l)=\frac{-16 H}{y_{f}}\left[\frac{l}{3}\left(\alpha^{3}-\beta^{3}\right)-\frac{1}{5}\left(\alpha^{5}-\beta^{5}\right)\right]+\frac{4 C}{y_{f}}\left[\frac{2 y_{f}}{3}\left(\gamma^{3}-\alpha^{3}\right)-4\left(\frac{l}{3}\left(\gamma^{3}-\alpha^{3}\right)-\frac{1}{5}\left(\gamma^{5}-\alpha^{5}\right)\right)\right] \\
& \frac{-4 H}{y_{f}}\left[\frac{4 y_{f}}{3}\left(\gamma^{3}-\alpha^{3}\right)-4\left(\frac{l}{3}\left(\gamma^{3}-\alpha^{3}\right)-\frac{1}{5}\left(\gamma^{5}-\alpha^{5}\right)\right)\right]+4\left[\frac{2 B}{5}\left(\delta^{5}-\gamma^{5}\right)-\frac{2\left(B\left(l-y_{f}\right)+C\right)}{3}\left(\delta^{3}-\gamma^{3}\right)\right] \\
& +4\left[\frac{-2 B}{5} \delta^{5}+\frac{2\left(B\left(l-y_{f}\right)-D\right)}{3} \delta^{3}\right]
\end{aligned}
$$




$$
D=\frac{3}{8(l-\lambda)^{\frac{3}{2}}}\left[\frac{4\left(y_{r}-l\right)}{y_{f}}\left(h 1\left(y_{r}-l\right)-h 2\right) A 1+\frac{c 1\left(y_{r}-l\right)-c 2}{c 3\left(y_{r}-l\right)-y_{f}}\left(y_{r}-l\right)\left(A 2-\frac{8}{3}\left((l-\lambda)^{\frac{3}{2}}-k 1\right)\right)-\frac{\beta W}{\rho U^{2}}\right]
$$

where functions $h 1, h 2, A 1, A 2, c 1, c 2, c 3, k 1$ are functions of known values and have been clumped together for equation simplification. Similarly, integral equations [6 and 11 can be split into four intervals and integration can be performed. Specifically, equation [1] would yield equation [26] using the symbols specified in equation 23

$$
\begin{aligned}
& x_{p}-\tan ^{-1}\left(x_{p}\right)=\frac{1}{(2 C+2 D)\left(\sqrt{y_{r}-l}\right)}\left[\frac { 4 H } { y _ { f } } \left[\frac{1}{3}\left(\alpha^{3}-\beta^{3}\right)-y_{r}(\sqrt{\alpha}-\sqrt{\beta})+y_{r} \sqrt{y_{r}-l}\left(\tan ^{-1}\left(\frac{\alpha}{\sqrt{y_{r}-l}}\right)\right.\right.\right. \\
& \left.\left.-\tan ^{-1}\left(\frac{\beta}{\sqrt{y_{r}-l}}\right)\right)\right]+\frac{4 C}{y_{f}}\left[\frac{1}{3}\left(\gamma^{3}-\alpha^{3}\right)-y_{r}(\sqrt{\gamma}-\sqrt{\alpha})+y_{r} \sqrt{y_{r}-l}\left(\tan ^{-1}\left(\frac{\gamma}{\sqrt{y_{r}-l}}\right)-\tan ^{-1}\left(\frac{\alpha}{\sqrt{y_{r}-l}}\right)\right)\right] \\
& +2 C\left[(\sqrt{\gamma}-\sqrt{\alpha})-\sqrt{y_{r}-l}\left(\tan ^{-1}\left(\frac{\gamma}{\sqrt{y_{r}-l}}\right)-\tan ^{-1}\left(\frac{\alpha}{\sqrt{y_{r}-l}}\right)\right)\right]-\frac{4 H}{y_{f}}\left[\frac{1}{3}\left(\gamma^{3}-\alpha^{3}\right)-y_{r}(\sqrt{\gamma}-\sqrt{\alpha})\right. \\
& \left.+y_{r} \sqrt{y_{r}-l}\left(\tan ^{-1}\left(\frac{\gamma}{\sqrt{y_{r}-l}}\right)-\tan ^{-1}\left(\frac{\alpha}{\sqrt{y_{r}-l}}\right)\right)\right]-4 H\left[(\sqrt{\gamma}-\sqrt{\alpha})-\sqrt{y_{r}-l}\left(\tan ^{-1}\left(\frac{\gamma}{\sqrt{y_{r}-l}}\right)\right.\right. \\
& \left.\left.-\tan ^{-1}\left(\frac{\alpha}{\sqrt{y_{r}-l}}\right)\right)\right]+\left(2 B y_{f}-2 C\right) \sqrt{y_{r}-l}\left(\tan ^{-1}\left(\frac{\sqrt{\gamma}}{\sqrt{y_{r}-l}}\right)-\sqrt{\gamma}\right)+2 B\left(y_{r} \sqrt{\gamma}-\frac{\gamma^{3}}{3}\right. \\
& \left.\left.-y_{r} \sqrt{y_{r}-l} \tan ^{-1}\left(\frac{\sqrt{\gamma}}{\sqrt{y_{r}-l}}\right)\right)+\pi \sqrt{y_{r}-l}\left(S\left(y_{r}-l\right)+B\left(l-y_{f}\right)-D\right)\right]
\end{aligned}
$$

where

$$
x_{p}=\sqrt{\frac{l-\lambda}{y_{r}-l}}
$$

Equation [6] would result in equation 29 based on the symbols provided in equation 28

$$
\begin{aligned}
& \beta=\sqrt{\frac{y_{r}-l}{l}}, \alpha=\sqrt{\frac{y_{r}-l}{l-\frac{y_{f}}{2}}}, \gamma=\sqrt{\frac{y_{r}-l}{l-y_{f}}}, \delta=\sqrt{\frac{y_{r}-l}{l-\lambda}} \\
& \tan ^{-1}\left(\frac{1}{x_{p}}\right)\left(1+x_{p}^{2}\right)+x_{p}=\frac{1}{(C+D)\left(y_{r}-l\right)}\left[\frac { 4 H ( y _ { r } - l ) } { y _ { f } } \left(\frac{l}{2}\left(\tan ^{-1}(\beta)\left(1+\frac{1}{\beta^{2}}\right)+\frac{1}{\beta}-\tan ^{-1}(\alpha)\left(1+\frac{1}{\alpha^{2}}\right)-\frac{1}{\alpha}\right)\right.\right. \\
& -\frac{\left(y_{r}-l\right)}{4}\left(\tan ^{-1}(\alpha)\left(1-\frac{1}{\alpha^{4}}\right)+\frac{1}{\alpha}\left(1-\frac{1}{3 \alpha^{2}}\right)-\tan ^{-1}(\beta)\left(1-\frac{1}{\beta^{4}}\right)-\frac{1}{\beta}\left(1-\frac{1}{3 \beta^{2}}\right)\right) \\
& +\frac{C}{y_{f}}\left(\left(2 l-y_{f}\right)\left(y_{r}-l\right)\left(\tan ^{-1}(\alpha)\left(1+\frac{1}{\alpha^{2}}\right)+\frac{1}{\alpha}-\tan ^{-1}(\gamma)\left(1+\frac{1}{\gamma^{2}}\right)-\frac{1}{\gamma}\right)\right. \\
& \left.-\left(y_{r}-l\right)\left(y_{r}-l\right)\left(\tan ^{-1}(\gamma)\left(1-\frac{1}{\gamma^{4}}\right)+\frac{1}{\gamma}\left(1-\frac{1}{3 \gamma^{2}}\right)-\tan ^{-1}(\alpha)\left(1-\frac{1}{\alpha^{4}}\right)-\frac{1}{\alpha}\left(1-\frac{1}{3 \alpha^{2}}\right)\right)\right) \\
& -\frac{H}{y_{f}}\left(\left(2 l-2 y_{f}\right)\left(y_{r}-l\right)\left(\tan ^{-1}(\alpha)\left(1+\frac{1}{\alpha^{2}}\right)+\frac{1}{\alpha}-\tan ^{-1}(\gamma)\left(1+\frac{1}{\gamma^{2}}\right)-\frac{1}{\gamma}\right)-\left(y_{r}-l\right)\left(y_{r}-l\right)\left(\tan ^{-1}(\gamma)\left(1-\frac{1}{\gamma^{4}}\right)\right.\right. \\
& \left.\left.+\frac{1}{\gamma}\left(1-\frac{1}{3 \gamma^{2}}\right)-\tan ^{-1}(\alpha)\left(1-\frac{1}{\alpha^{4}}\right)-\frac{1}{\alpha}\left(1-\frac{1}{3 \alpha^{2}}\right)\right)\right)+\left(y_{r}-l\right)(B(l-y f)+C)\left(\tan ^{-1}(\beta)\left(1+\frac{1}{\beta^{2}}\right)+\frac{1}{\beta}\right) \\
& +\frac{B}{2}\left(y_{r}-l\right)\left(y_{r}-l\right)\left(\tan ^{-1}(\gamma)\left(1-\frac{1}{\gamma^{4}}\right)+\frac{1}{\gamma}\left(1-\frac{1}{3 \gamma^{2}}\right)\right)-\frac{\pi B}{4}\left(y_{r}-l\right)\left(y_{r}-l\right) \\
& \left.-\frac{\pi}{2}\left(y_{r}-l\right)\left(B\left(l-y_{f}\right)-D\right)+\frac{\pi}{4}\left(y_{r}-l\right)\left(2 B\left(l-y_{f}\right)-2 D+S\left(y_{r}-l\right)\right)\right]
\end{aligned}
$$

There are two unknowns, $\lambda$ and $y_{r}$ and two equations [26] and 29] In order to do this, the optimization problem is recast such that the squared difference of the right hand side and left hand side of the equation 26] is minimized while equation 29] is used as an equality constraint. 


\section{Meta-model estimation of minimum area distribution}

An approximate analysis is sought for the solution of the SGD equations. The procedure laid out in the previous section can be used effectively to estimate the area distributions for minimum boom footprints. However, it has been observed that once in a while the optimization terminates prematurely. Even if it does converge to the right solution, the numerical integration and optimization routines could take about 2-3 seconds. That time is a lot if the analysis has to be run multiple times as in an optimization study. Therefore, a non-linear approximation to the SGD solution procedure is pursued. Since the responses are non-linear with respect to the inputs, a neural network meta-model has been used.

An artificial neural network with a single hidden layer is a universal approximator to any function provided the right number of neurons are chosen in the hidden layer $\stackrel{20}{20}$ For a single hidden layer neural network, the outputs can be specified in terms of the inputs by an equation such as the one shown in equation [30. The $\sigma$ in this equation represents a non-linear sigmoidal transfer function, usually with activation 1.0.

$$
y=W^{T} \sigma\left(V^{T} X+b_{1}\right)+b_{2}
$$

Supervised networks with batch training are used in this study. Supervised neural networks have two stages. The first is the training stage where the actual analysis is run to record the input-output combinations. This data is then fed to the network which changes the weight matrices and bias vectors to fit the data in the best possible way. There are various algorithms to fit the data. In this study, bayesian regression learning algorithm available in the MATLAB neural network ${ }^{21}$ toolbox is used. Once the optimum weight and bias vectors are obtained, the model has to be tested for performance. A test data of input-output pairs is generated using the actual analysis and it is compared with the output from neural network metamodel. If the neural network predicts the test data set satisfactorily, one can assume that the neural network has successfully approximated the analysis function. A training and test data for using the SGD solution procedure has been created with the ranges for the variables provided in table 4

\begin{tabular}{|c|c|c|}
\hline Variable & Lower bound & Upper bound \\
\hline B (Slope in F-function) & 0.0 & 0.0004 \\
\hline Mach Number & 1.4 & 1.8 \\
\hline Length & $100.0 \mathrm{ft}$ & $200.0 \mathrm{ft}$ \\
\hline Gross Weight & $80000.0 \mathrm{lbs}$ & $130000.0 \mathrm{lbs}$ \\
\hline Altitude & $50000.0 \mathrm{ft}$ & $80000.0 \mathrm{ft}$ \\
\hline$y_{f}$ (Bluntness parameter) & 2.0 & 30.0 \\
\hline
\end{tabular}

Table 4. Ranges for SGD input variables

A network with 18 hidden layers was chosen. This number was chosen by trial and error to obtain the best possible fit. Figure 3 compares the actual training data with those obtained from the neural network. It can be seen from this figure that the neural network was able to successfully track the actual responses by modifying the weights and biases. A good match with training data is only half the story. The most important thing is that the network has to perform well for the test data. Figure 4 compares the actual test data with the output from the neural network. From this figure, it can be concluded that the trained neural network can be used as a viable replacement to the SGD analysis.

\section{Coarse-grained parallel genetic algorithm}

Most computational simulations these days are being run simultaneously on multiple computers. This would enable most computationally intensive tasks like CFD simulations or genetic algorithm shape optimization runs to be completed much faster, provided enough computers are present in the cluster and computational decomposition and communication between processors is handled well. Parallelization is used in this study to expedite the turn around time of the analyses.

Genetic algorithms have a few important advantages over gradient based optimization schemes. Firstly, they achieve a global optimum instead of getting stuck in a local optimum. Secondly, since they operate on 

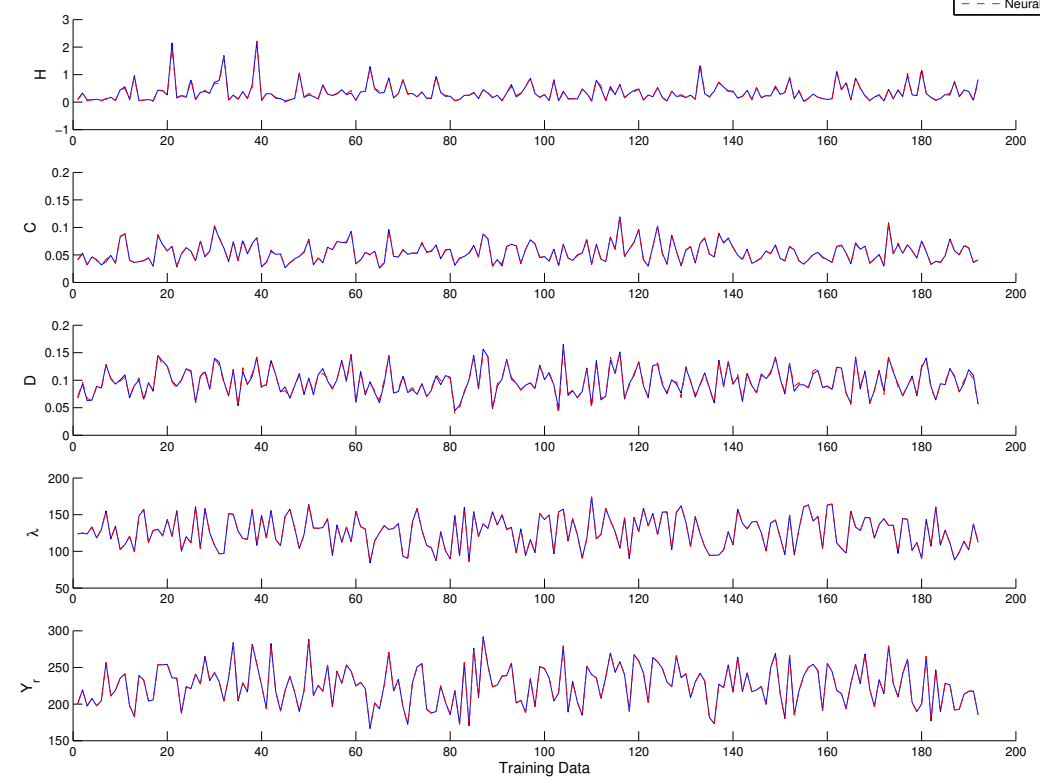

Figure 3. Neural Network training for SGD equations
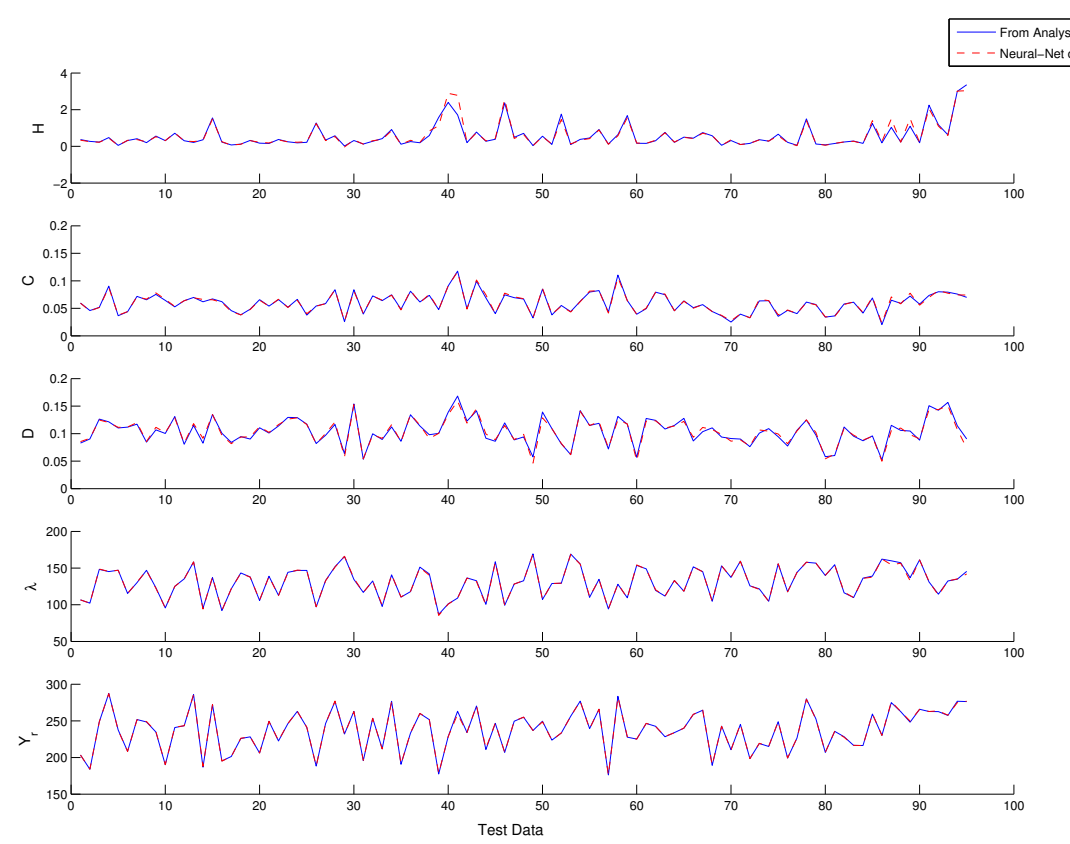

Figure 4. Neural Network testing for SGD equations 
population of candidates, a Pareto-optimal front can be obtained in a multi-dimensional space with many conflicting objective functions. Furthermore, since these do not require any gradient information, they can be applied to problems which may be discontinuous. In spite of these advantages, genetic algorithms have been the subject of criticism for various reasons. They are very computationally intensive as they lack the elegance of reaching the optimum as in the case of gradient based optimization. Secondly, as the algorithm continues, some individuals with high fitness values may dominate the population. This causes premature convergence of the population and should be avoided.

Premature convergence is avoided in most genetic algorithms using a technique called niching $\stackrel{22]}{2}$ which tries to include a diverse population after every generation or epoch. A niching genetic algorithm has the ability to include a diverse population at each generation. However, an efficient parallel implementation of the genetic algorithm could obtain the results in far less computational time. Various parallelization schemes have been proposed including those by Gondra 23 and de Toro ${ }^{24}$ In this study, a parallel genetic algorithm is attempted along the lines suggested by Gondra. The basic genetic algorithm is the Non-dominated sorting genetic algorithm (NSGA2) proposed by Deb!25

A coarse grained genetic algorithm is based on the principle of punctuated equilibria, which is based on allopatric speciation and stasis. Any population initially undergoes rapid evolution to new population. However, as the number of generations increase, the rate of evolution decreases and the changes to the population are gradual and slow. In that sense, the population attains stability or stasis and could end up in a local optimum or prematurely converged. Punctuated equilibrium principle states that in order to continue the evolution to the best population, new population members have to be thrust into the existing stable population to increase the evolution rate. Allopatric speciation involves the introduction of stabilized individuals into different populations. The implementation of this is shown in the final results.

\section{Shape Optimization results}

To numerically minimize sonic boom loudness, a 'bi-level reverse' optimization is performed. The analysis is split into two optimization routines. Firstly, using probabilistic propagation techniques, the optimum area distribution, aircraft length, Gross Weight, Mach number and altitude which minimize the perceived loudness level on the ground are determined. This optimum distribution is then fed to the next optimization level, where optimum shape parameters, described in section II, are obtained to match the area distribution. The following sections briefly explain these steps and provide the shape optimization results.

\section{A. Optimum area distribution}

The design variables in this step are the Mach number, gross weight, length, altitude, bluntness parameter $y_{f}$ and slope of the rise in F-function, B. Using the neural network meta-model, optimum values for these variables are obtained by simultaneously minimizing the probabilistic estimate of the perceived loudness and maximizing the figure of merit ${ }^{26}$ as defined in equation 31 The reason for providing the second conflicting objective is to obtain a Pareto-front of area distributions. The best compromised area distribution can then be chosen according to the requirements of the design.

$$
F o M=\frac{\beta W}{P_{g} l^{\frac{3}{2}} \sqrt{h}} e^{\frac{h}{2 H}} 10^{3}
$$

where $H$ is the atmospheric scale height.

The ranges of the design variables are shown in table 5 Note that these ranges are a subset of the ranges utilized for the neural network and can be safely used as a replacement to the actual analysis. The results for the first step of optimization are shown in figures 5 and [6 Figure [5] shows the Pareto-front of the probabilistic perceived loudness level against the inverse of figure of merit. The genetic algorithm was run for 30 generations.

Figure [6] shows the target equivalent area distribution to be used for the second optimization step. The final values chosen for the second step of optimization are $\mathrm{M}=1.44, \mathrm{GW}=113401.76 \mathrm{lbs}$, Altitude $=59501.73 \mathrm{ft}$, length $=149.54 \mathrm{ft}$. 


\begin{tabular}{|c|c|c|}
\hline Design Variable & Lower bound & Upper bound \\
\hline B (Slope in F-function) & 0.0 & 0.0004 \\
\hline Mach Number & 1.4 & 1.8 \\
\hline Length & $100.0 \mathrm{ft}$ & $150.0 \mathrm{ft}$ \\
\hline Gross Weight & $100000.0 \mathrm{lbs}$ & $130000.0 \mathrm{lbs}$ \\
\hline Altitude & $50000.0 \mathrm{ft}$ & $60000.0 \mathrm{ft}$ \\
\hline$y_{f}$ (Bluntness parameter) & 2.0 & 30.0 \\
\hline
\end{tabular}

Table 5. Ranges of design variables for step 1

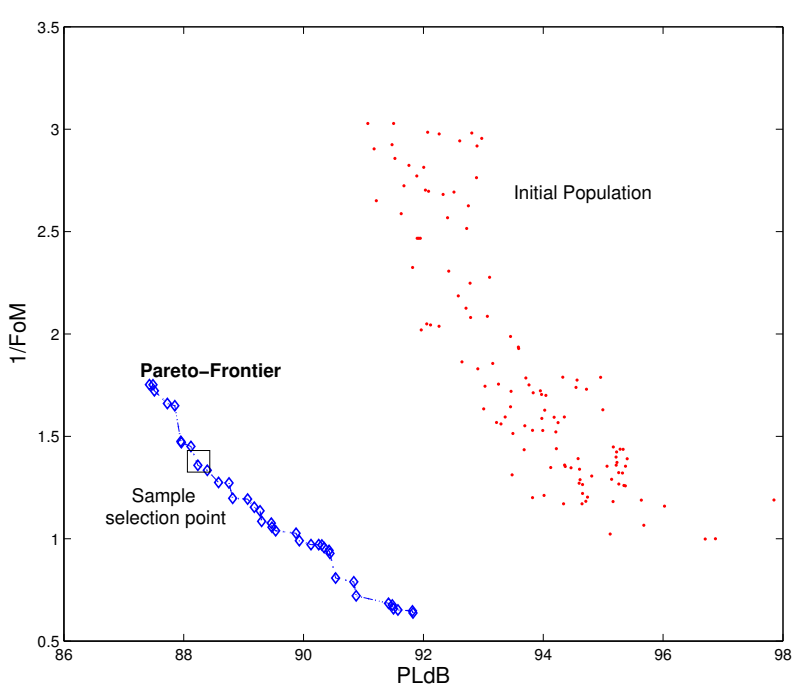

Figure 5. Pareto-front for the first step of optimization

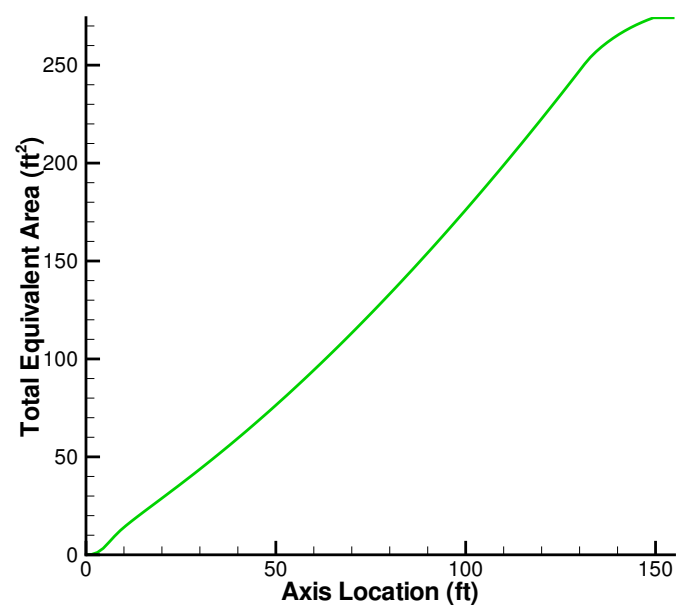

Figure 6. The target equivalent area distribution chosen for step2 


\section{B. Estimation of optimum aircraft shape}

The design variables in this step are the shape parameters of the aircraft. Suitable care is taken to include a vast design space for the aircraft shapes to achieve a proper final shape. A parallel genetic algorithm is utilized to minimize the normalized squared difference between the total equivalent area from the aircraft and the target area distribution. After about 20 generations, figure $\mathbf{7}$ depicts the comparison of the total equivalent areas. As can be observed from the figure, a close match is obtained for most of the longitudinal locations. The comparison is not as good for the tail regions. The reason for this could be that there is not sufficient shape control in the tail sections of the aircraft.

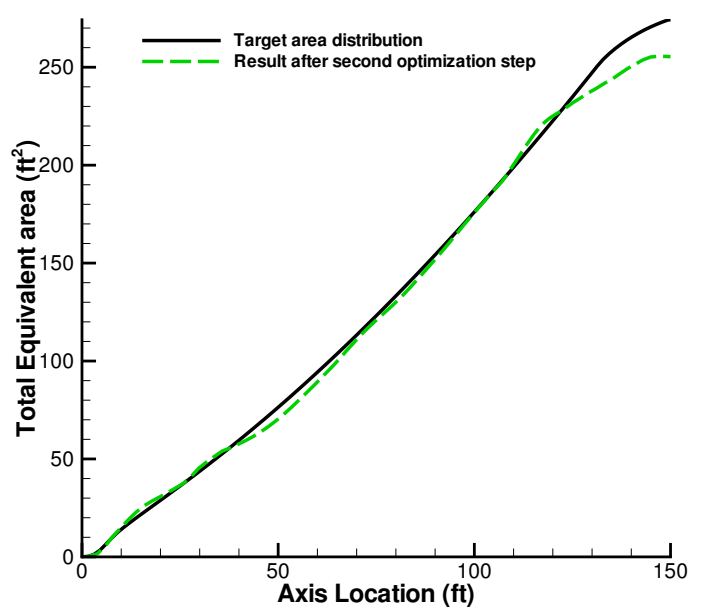

Figure 7. Comparison of total equivalent areas

Figure 8 depicts the Pareto-front for the optimization run. The trade-off between boom minimization and aircraft performance is seen in this figure, although the measure for boom minimization has been mapped from the usual loudness level to the matching of the total equivalent areas. The dashed rectangle provides the best candidates which offer a fair compromise between sonic boom and performance constraints. One such candidate configuration is provided in figure 9

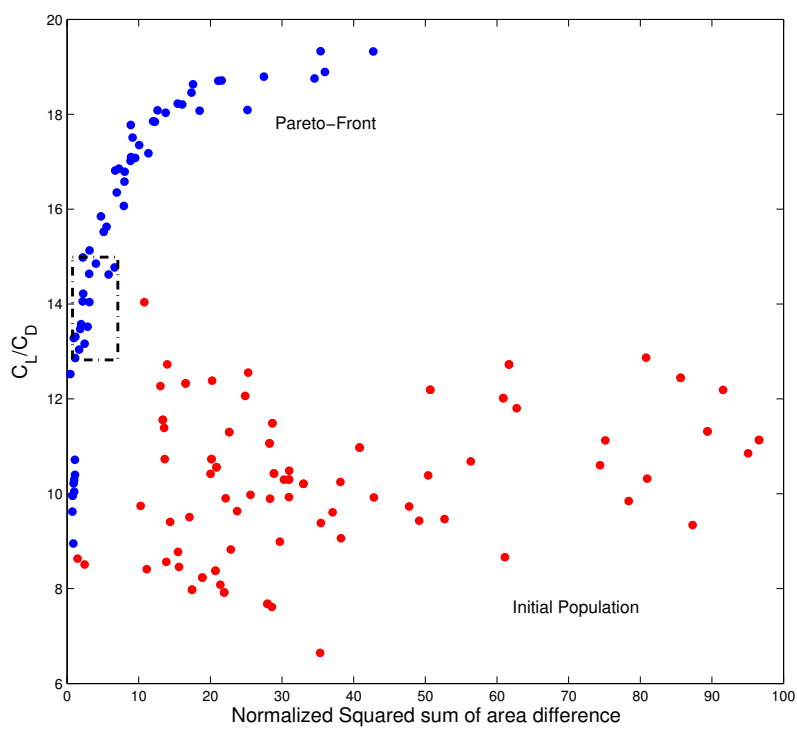

Figure 8. Pareto-front for the second step of optimization 




Figure 9. One of the best candidate configurations

It is important to measure the performance of the parallel genetic algorithm. Figure 10 depicts the superposition of a linear speed-up and the speed-up achieved by the proposed genetic algorithm. As can be seen from the figure, a sub-linear speed-up is achieved. It might be possible to improve the speed-up by better communication calls between processors.

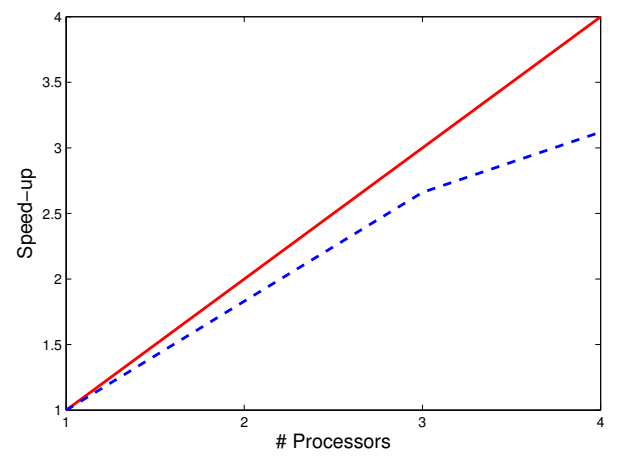

Figure 10. Speed-up of the Parallel GA

\section{Conclusions}

A new design methodology has been introduced for sonic boom minimization based on linearized methods. The geometry generation and discretization procedure enables an efficient and automatic way to combine linearized and non-linear analysis. The unique shape optimization procedure in conjunction with parallel genetic algorithms allows the designer the explore vast design spaces efficiently and accurately. The probabilistic propagation provides a strategy to include atmospheric fluctuations into the aircraft design process. The bi-level procedure not only serves as a pseudo-inverse technique but also induces design flexibility by separating the near and far field analysis.

\section{Acknowledgements}

Geometry generation and discretization part of the work was supported under grant NAG-1-02023, "Design Methodology for Revolutionary Aerospace Concepts", from NASA Langley Research Center with 
Mr.Craig Nickol as the technical monitor. The research on configurations for low boom was funded under grant NIA 3003-GT "Configuration Optimization for low boom". We would like to express our sincere thanks to Mr.Suraj Unnikrishnan for help with the Neural Network architecture implementation. Michael Buonanno's help with the MATLAB geometry generator is appreciated.

\title{
References
}

\begin{abstract}
${ }^{1}$ Mavris, D. N. and Delaurentis, D., "Methodology for examining the simultaneous impact of requirements, vehicle characteristics, and technologies on military aircraft design," 22nd Congress of the International Council on the Aeronautical Sciences, ICAS, Aug. 2000.

${ }^{2}$ Darden, C. M., "The importance of sonic boom research in the development of future high speed aircraft," Journal of the NTA, 1992, pp. 54-62.

${ }^{3}$ Seebass, R., "History and economics of, and prospect for, commercial supersonic transport," RTO AVT course on Fluid dynamics research on supersonic aircraft, RTO, May 1998.

${ }^{4}$ Bloor, M. I. and Wilson, M. J., "Efficient parameterization of generic aircraft geometry," Journal of Aircraft, Vol. 32, No. 6, Dec. 1995, pp. 1269-1275.

${ }^{5}$ Robert E. Smith, Malcolm I.G. Bloor, M. J. W. and Thomas, A. M., "Rapid Airplane Parametric Input Design," Proceedings of the 12th AIAA Computational Fluid dynamics conference, American Institute for Aeronautics and Astronautics, June 1995.

${ }^{6}$ Patricia A. Kerr, R. E. S. and Posenau, M.-A. K., "GEOLAB Surface modeling and grid generation technology and services," Tech. Rep. NASA 95-CP3291, NASA Langley Research Center, Hampton, VA, May 1995.

${ }^{7}$ Samareh, J. A., "A novel shape parameterization approach," Tech. Rep. NASA TM-1999-209116, NASA Langley Research Center, Hampton, VA, May 1999.

${ }^{8}$ Samareh, J. A., "Survey of shape parameterization techniques for high-fidelity multidisciplinary shape optimization," AIAA Journal, Vol. 39, No. 5, May 2001, pp. 877-884.

${ }^{9}$ Rallabhandi, S. K. and Mavris, D. N., "Sonic Boom modelling under atmospheric uncertainty," Proceedings of the 10th AIAA/ISSMO Multidisciplinary Analysis and Optimization Conference, American Institute for Aeronautics and Astronautics, Aug. 2004.

${ }^{10}$ Carlson, H. and Maglieri, D., "Review of sonic boom generation theory and prediction methods," Journal of Acoustical Society of America, Vol. 51, No. 2, 1972, pp. 675-685.

${ }^{11}$ Harris, R. V., "An analysis and correlation of Aircraft wave drag," Tech. Rep. NASA TM X-947, NASA, Princeton,NJ, April 1964.

${ }^{12}$ Middleton, W. D. and Carlson, H. W., "Numerical method for estimation and optimizing supersonic aerodynamic characteristics of arbitrary planform wings," Journal of Aircraft, Vol. 2, No. 4, July 1965, pp. 261-265.

${ }^{13}$ Rallabhandi, S. K. and Mavris, D. N., "An unstructured wave drag code for preliminary design of future supersonic aircraft," Proceedings of the 33rd Fluid dynamics conference and exhibit, American Institute for Aeronautics and Astronautics, June 2003.

${ }^{14}$ Wallace D. Hayes, R. C. H. and Kulsrud, H. E., "Sonic boom propagation in a stratified atmosphere with computer program," Tech. Rep. NASA CR-1299, Aeronautical research associates of Princeton Inc., Princeton,NJ, April 1969.

${ }^{15}$ Plotkin, K. J., "PCBoom3 Sonic boom prediction model - Version 1.0c," Tech. Rep. AFRL-HE-WP-TR-2001-0155, Wyle
\end{abstract} Research laboratories, Arlington,VA, May 1996.

16 "Anderson-Darling: A Goodness of Fit Test for small Samples Assumptions," http://rac.alionscience.com/pdf/A_DTest.pdf

${ }^{17}$ Seebass, R. and George, A., "Sonic boom minimization," Journal of Acoustical Society of America, Vol. 51, No. 2, 1972, pp. 686-694.

${ }^{18}$ Darden, C., "Sonic boom minimization with nose-bluntness relaxation," Tech. Rep. NASA TP-1348, NASA Langley Research Center, Hampton, VA, Sept. 1979.

${ }^{19}$ Leatherwood, J. D. and Sullivan, B. M., "Effect of sonic boom asymmetry on subjective loudness," Tech. Rep. TM-107708, National Aeronautics and Space Administration, Langley, Virginia, Dec. 1992.

${ }^{20}$ Haykin, S., NEURAL NETWORKS A Comprehensive Foundation, Pearson Education, 1999.

${ }^{21}$ MathWorks, "Neural Network toolbox user's guide," http://www.mathworks.com/access/helpdesk/help/toolbox/nnet/ 2002.

${ }^{22}$ Srinivas, N. and Deb, K., "Multiobjective Optimization using nondominated sorting in genetic algorithms," Journal of Evolutionary Computation, Vol. 2, No. 3, Jan. 1995, pp. 221-248.

${ }^{23}$ Gondra, I. and Samadzadeh, M. H., "A coarse-grain parallel genetic algorithm for finding Ramsey numbers," Proceedings of the 18th Annual ACM Symposium on Applied Computing, March 2003.

${ }^{24}$ Francisco de Toro, Julio Ortega, J. F. and Diaz, A., "PSFGA: A Parallel Genetic Algorithm for Multiobjective Optimization," Proceedings of the 10th Euromicro workshop on Parallel, Distributed and Network-based Processing, IEEE, June 2002.

${ }^{25}$ Kalyanmoy Deb, Amrit Pratap, S. A. and Meyarivan, T., "A fast and elitist multi-objective genetic algorithm: NSGA-II," Tech. Rep. KanGAL report 200001, IIT Kanpur, Kanpur, India, 2000.

${ }^{26}$ Seebass, R. and Argrow, B., "Sonic boom minimization revisited," Proceedings of the 2nd Theoretical Fluid Mechanics Meeting, American Institute for Aeronautics and Astronautics, June 1998. 